\title{
Filosofía y conductismo. Una relación lógica, ontológica, epistemológica y existencial
}

\author{
Philosophy and behaviorism. A logical, ontological, epistemological and \\ existential relationship
}

\section{Filosofia e behaviorismo. Uma relação lógica, ontológica, epistemológica e} existencial

\author{
José Nava Bedolla \\ Instituto Superior de Ciencias de la Educación del Estado de México, México \\ nava5812@yahoo.com.mx \\ https://orcid.org/0000-0003-4378-9673
}

\section{Resumen}

El objetivo central de la presente investigación consistió en analizar el tipo de relaciones que se pueden establecer entre la filosofía y la teoría conductista aplicada a la educación. Se partió del supuesto de que las mentadas relaciones se establecen en el sentido de que el motor que promueve los principios de la ciencia o del conocimiento que se utilizan para elaborar los discursos de la teoría conductista son las necesidades existenciales, epistemológicas, ontológicas y lógicas del sujeto cognoscente dependiendo de circunstancias de modo, tiempo y lugar.

Se trató de un estudio de tipo metacientífico, es decir, epistemológico. Se pretendió hacer ciencia de la ciencia o, lo que es lo mismo, conocimiento del conocimiento para apreciar los alcances y limitaciones de la teoría conductista aplicada a la educación en relación con la idea de filosofía. En un primer movimiento, se reelaboró la "definición” de la categoría denominada con la palabra filosofía. En segundo lugar, se reconstruyó el mapeo de la idea enunciada con el término teoría conductista. Finalmente, se establecieron las relaciones entre la filosofía y la teoría conductista, a partir de las necesidades existenciales del sujeto cognoscente. 


\section{Revista Iberoamericana \\ de las Ciencias Sociales y Humanísticas}

ISSN: $2395-7972$

Los resultados revelan la existencia de una relación o correspondencia entre la filosofía y la teoría conductista aplicada a la educación en términos de necesidades existenciales, epistemológicas, ontológicas y lógicas del sujeto cognoscente. Lo anterior permite concluir, entre otras cosas, que el mapeo del concepto mencionado con la voz conductismo se establece en términos de alguna definición de la noción mentada con la voz filosofía que determina la utilización de ciertos principios del conocimiento o de la ciencia dependiendo de las necesidades lógicas, ontológicas, epistemológicas y existenciales del sujeto cognoscente de la teoría conductista. Así, es posible sospechar que toda teoría, además de no ser neutral, está determinada por las necesidades mencionadas.

Palabras clave: conductismo, epistemológicas, filosofía, necesidades existenciales, ontológicas y lógicas, principios de la ciencia o del conocimiento.

\footnotetext{
Abstract

The main objective of this research was to analyze the type of relationships that can be established between philosophy and behavioral theory applied to education.

It was started from the assumption that the aforementioned relationships are established in the sense that the engine that promotes the principles of science or knowledge that are used to elaborate the discourses of behaviorist theory are the existential, epistemological, ontological and logical needs of the knowing subject depending on circumstances of mode, time and place.

It was a meta-scientific study, that is, epistemological. It was intended to make science of science, or what is the same, knowledge of knowledge; to appreciate the scope and limitations of the behavioral theory applied to education in relation to the idea of philosophy.

In a first movement, the definition of the category named with the word $<<$ philosophy $>>$ is reworked.

Second, the mapping of the stated idea is reconstructed with the term "behaviorist theory". Finally, the <<relations >> between philosophy and behaviorist theory are established, based on the existential needs of the knowing subject.

The results reveal the existence of a relationship or correspondence between philosophy and behaviorist theory applied to education in terms of existential, epistemological, ontological and logical needs of the knowing subject.
} 


\section{Revista Iberoamericana de las Ciencias Sociales y Humanísticas}

ISSN: 2395 - 7972

The above allows to conclude, among other things, that the mapping of the concept mentioned with the voice <<behaviorism>> is established in terms of some definition of the notion mentioned with the voice $<<$ philosophy $\gg$ that determines the use of certain principles of knowledge or science depending on the logical, ontological, epistemological and existential needs of the knowing subject of the behaviorist theory.

Then then, it is possible to suspect that every theory, in addition to being neutral, is determined by the needs mentioned in the preceding paragraph.

Keywords: behaviorism, epistemological, philosophy, existential, ontological and logical needs, principles of science or knowledge.

\section{Resumo}

O objetivo principal desta pesquisa foi analisar o tipo de relações que podem ser estabelecidas entre a filosofia e a teoria comportamental aplicada à educação.

Partiu-se do pressuposto de que as referidas relações se estabelecem no sentido de que o motor que promove os princípios da ciência ou do conhecimento que se utilizam para elaborar os discursos da teoria behaviorista são as necessidades existenciais, epistemológicas, ontológicas e lógicas dos conhecer o assunto dependendo das circunstâncias do modo, hora e lugar.

Foi um estudo meta-científico, ou seja, epistemológico. Pretendia-se fazer ciência da ciência, ou seja, conhecimento do conhecimento; apreciar o alcance e as limitações da teoria comportamental aplicada à educação em relação à ideia de filosofia.

Em um primeiro movimento, a definição da categoria nomeada com a palavra $<<$ filosofia >> é retrabalhada.

Em segundo lugar, o mapeamento da ideia declarada é reconstruído com o termo "teoria behaviorista".

Finalmente, as <<relações>> entre filosofia e teoria behaviorista são estabelecidas, a partir das necessidades existenciais do sujeito cognoscente.

Os resultados revelam a existência de uma relação ou correspondência entre filosofia e teoria behaviorista aplicada à educação em termos de necessidades existenciais, epistemológicas, ontológicas e lógicas do sujeito que conhece.

$\mathrm{O}$ exposto permite concluir, entre outras coisas, que o mapeamento do conceito mencionado com a voz <<behaviorismo>> é estabelecido em termos de alguma definição da noção 


\section{Revista Iberoamericana de las Ciencias Sociales y Humanísticas}

mencionada com a voz $\ll$ filosofia $\gg$ que determina o uso de certos princípios da conhecimento ou ciência dependendo das necessidades lógicas, ontológicas, epistemológicas e existenciais do sujeito cognoscente da teoria behaviorista.

Então, é possível suspeitar que toda teoria, além de neutra, é determinada pelas necessidades mencionadas no parágrafo anterior.

Palavras-chave: behaviorismo, epistemológicas, filosofia, necessidades existenciais, ontológicas e lógicas, princípios da ciência ou do conhecimento.

Fecha Recepción: mayo 2020

Fecha Aceptación: diciembre 2020

\section{Introducción}

Se dice que la teoría no es neutral, pues obedece a determinados intereses subjetivos o grupales de quienes construyen las ideas, y el conductismo - como una gran teoría que se puede aplicar a la educación - no escapa a dichos juicios. De hecho, también se argumenta que la teoría depende de la filosofía, pero cómo se puede interpretar esta idea. Tomando en consideración esto, la pregunta de investigación que dio origen al presente trabajo quedó formulada de la siguiente manera: ¿cuáles son las relaciones que se pueden establecer entre la filosofía y la teoría conductista aplicada a la educación? La respuesta tentativa podría ser que entre la filosofía y la teoría se pueden establecer una serie de relaciones: la principal de ellas sería que la segunda depende de la primera, es decir, entre la filosofía y la teoría se puede establecer una relación de dependencia de la segunda respecto de la primera. Derivado de la asociación anterior, se propusieron otros vínculos, como que entre la filosofía y la teoría se pueden observar relaciones lógicas, ontológicas, epistemológicas y existenciales.

Con el propósito de demostrar las hipótesis anteriores, se reconstruyó —en primer lugar - una definición del concepto filosofía, para lo cual se tomaron algunas de las más importantes elaboradas a lo largo de la historia. La metodología empleada fue la siguiente: se enunciaron algunas definiciones; después se trató de explorar, describir, explicar, interpretar y comprender sus sentidos y significados; posteriormente se adoptó una postura frente a ellas; luego se justificó en términos existenciales, epistemológicos, ontológicos y lógicos; a continuación se aclaró el término; subsiguientemente se justificó, y finalmente se analizó y sintetizó. 


\section{Revista Iberoamericana de las Ciencias Sociales y Humanísticas}

ISSN: 2395 - 7972

En un segundo momento, se reconstruyó el mapeo del concepto conductismo mediante el siguiente procedimiento: se determinaron las necesidades existenciales, epistemológicas, ontológicas y lógicas del sujeto cognoscente de la teoría conductista, y se establecieron los principios del conocimiento a partir de los cuales se intenta hacer ciencia de la conducta desde el conductismo aplicado a la educación.

Por último, se fijaron las "relaciones" lógicas, epistemológicas, ontológicas y existenciales entre la filosofía y la teoría conductista aplicada a la educación de la siguiente manera: se establecieron los principales problemas filosóficos del conocimiento de la conducta humana, se determinaron las facultades cognoscitivas con las que el sujeto cognoscente pretende resolver los grandes problemas filosóficos del conocimiento de la conducta humana en el conductismo, se fijaron sus necesidades existenciales y ontológicas, se esclarecieron los principios de la ciencia de los que parte para generar conocimiento y se determinaron los supuestos filosóficos en los que, de manera irremediable, se ubica al pretender hacer ciencia de la conducta.

Los resultados demuestran que se puede sugerir una relación o correspondencia entre la filosofía y la teoría conductista en términos de necesidades existenciales, epistemológicas, ontológicas y lógicas del sujeto cognoscente en determinado tiempo, lugar y circunstancias.

Es interesante señalar, derivado de las conclusiones de la investigación, que - con conocimiento o no del hecho - todo discurso teórico - $-\mathrm{y}$, en este caso, el conductismo aplicado a la educación no escapa a ello — se debe a determinadas necesidades existenciales, ontológicas, lógicas y epistemológicas del sujeto cognoscente que pretende construir conocimiento. Esto implica, entre otras cosas, que la ciencia no es neutral y que se debe preparar a nuestros futuros investigadores, que en estos momentos se encuentran estudiando posgrados en epistemología, para que no vayan "a ciegas" en la construcción de sus objetos de estudio. 


\section{La definición del concepto denominado con el término filosofía}

\section{Enunciación de algunas "definiciones" de la idea determinada con la expresión filosofía}

El concepto denominado con la expresión filosofía ha sido "definido" por distintos pensadores, intelectuales o filósofos de diferentes épocas y contextos, de ahí que se le hayan otorgado múltiples acepciones, como 'amor a la sabiduría', 'conocimiento obtenido a través de la iluminación o cercanía racional con Dios', 'autorreflexión del espíritu humano mediante los supremos valores teóricos y prácticos para llegar a la concepción del universo' o 'ciencia de todas las cosas por sus últimas causas', por mencionar algunas.

Algunos pensadores entienden el vocablo filosofía del siguiente modo: "la ciencia teórica de los primeros principios y de las primeras causas" (Aristóteles, 1992, p. 8); "es su esencia, cualquiera que sea el objeto inmediato de sus esfuerzos, mantenerse en la pura observación y el investigar, pero no el dar reglas" (Schopenhauer, 1997, p. 215); "se asemeja más a un organismo que a un conjunto, y también es más propio referirla a evolución que a composición" (Bergson, 1986, p. 33); "la ciencia fundamental (...), la ciencia que tiene que asegurar la unidad de conocimientos humanos por la demostración de un último fundamento común" (Natorp, 1987, p. 7); "intento del espíritu humano para llegar a una concepción del universo mediante la autorreflexión sobre sus funciones valorativas, teóricas y prácticas" (Hessen, 2009, p. 9); "un corresponder que traduce a lenguaje la llamada del ser del ente" (Heidegger, 2006, p. 66); "conocimiento del universo o de todo cuanto hay, pero al partir ni sabemos qué es lo que hay, ni si lo que hay forma universo o multiverso, ni si, universo o multiverso, será cognoscible" (Ortega y Gasset 1998, p. 40).

\section{Exploración, descripción, explicación, interpretación y comprensión de las "definiciones" de la noción designada con el vocablo filosofía}

Si se suman y se multiplican las coincidencias y se restan y dividen las divergencias entre los autores comentados en torno de la definición del concepto denominado con el término filosofía, es posible analizar y sintetizar la delimitación mencionada desde diferentes sentidos y significados mediante una serie de inducciones y deducciones, comparaciones y clasificaciones que puedan remitir a una "definición" que permita arribar a una toma de postura. Se le ha entendido en términos de "un sistema coherente de puntos de vista sobre el mundo (es decir: la naturaleza, la sociedad y el pensamiento) que influye de manera 


\section{Revista Iberoamericana de las Ciencias Sociales y Humanísticas}

fundamental en la actividad del hombre" (Hessen, 2009, p. 9); como la correspondencia que se puede establecer entre el ente y el ser a través del lenguaje (Heidegger, 2006, p. 66); también en el sentido de los primeros principios y las primeras causas de la ciencia, entendiendo por "principio..., en primer lugar, punto de partida de la cosa" (Aristóteles, 1992, p. 75), “aquello mediante lo que puede hacerse mejor una cosa, por ejemplo, el principio de una ciencia" (Aristóteles, 1992, p. 75), "la parte esencial y primera de donde proviene una cosa" (Aristóteles, 1992, p. 75) y, por otra parte, "se llama causa (Aristóteles, 1992, p. 76), ya la materia de que una cosa se hace", "primer principio del cambio o del reposo" (Aristóteles, 1992, p. 76), "aquello que se hace es causa de lo hecho, y lo que imprime el cambio lo es de lo que experimenta el cambio" (Aristóteles, 1992, p. 76), "todos los intermedios entre el motor y el objeto" (Aristóteles 1992, p. 76); por lo tanto, "la filosofía no puede hacer otra cosa que interpretar y explicar lo existente" (Schopenhauer, 1997, p. 215), en su determinación voluntaria de manera autónoma porque "la conducta del hombre y el mundo mismo son voluntad, sólo en ese supuesto es verdaderamente autónoma (Schopenhauer, 1997, p. 216); también se entiende como "la ciencia que pretende prescindir de símbolos" (Bergson, 1986, p. 7), y que "se asemeja más a un organismo que a un conjunto, y también es más propio referirla a evolución que a composición” (Bergson, 1986, p. 33); por otra parte es "conocimiento por principios, mas no se dirige a los objetos sino a la unidad del mismo conocimiento" (Natorp, 1987, p. XIV), porque "los objetos del conocimiento son inagotables y el sabio se acerca a ellos, pero nunca los alcanza totalmente" (Natorp, 1987, p. XIV), además “aunque es proceso infinito, el saber no está privado de la ley ni de la dirección, sólo gracias al método se avanza en la ciencia" (Natorp, 1987, p. XIV), por ello, "el ser es la eterna incógnita, que paso a paso el conocimiento va determinando mejor, pero el valor de la determinación depende exclusivamente del método de conocimiento, de su proceder, por ello, la propia filosofía es método" (Natorp, 1987, p. XIV), luego entonces "será la "verdadera filosofía' aquella que, por una parte, pueda considerarse como la base última de todo saber, por otra, que su alcance sea lo suficientemente amplio para que baste a la totalidad de los objetos que caen en el reino de todo conocimiento" (Natorp, 1987, p. 7), para cumplir con el primer requisito (ser la base última de todo saber), según Natorp (1987, p. 7), la filosofía debe ser una ciencia (criterio formal) y, para el segundo requisito (abarcar la totalidad de objetos que se puedan conocer), la filosofía necesita limitar los diferentes intereses del conocimiento (criterio material) debido a que "toda idea es pensada y todo cuadro es pintado 
Revista Iberoamericana

de las Ciencias Sociales y

Humanísticas

ISSN: $2395-7972$

desde ciertas suposiciones o convenciones tan básicas, tan de clavo pasado para el que pensó la idea o pintó el cuadro, que ni siquiera repara en ellas y por lo mismo no las introduce en su idea ni en su cuadro" (Ortega y Gasset, 1998, p. 21).

\section{Toma de partido sobre las "definiciones" del concepto nombrado con la palabra filosofía}

De la "definición” del concepto de filosofía elaborada por Hessen (2009, p. 9), conviene recuperar las ideas sobre las "funciones valorativas, teóricas y prácticas del alma humana", es decir, las necesidades que satisface la razón del sujeto cognoscente en una relación de conocimiento con su objeto de estudio son de conocimiento de la realidad (función teórica), de la estabilidad, permanencia o persistencia del ser humano en la misma (funciones prácticas) y de la forma de valorar las decisiones que se tomen sobre las dos anteriores (funciones valorativas). En Heidegger (2006, p. 66) se deben considerar las ideas de ente, en su sentido de "realidad" o "cosa", de ser en su concepción de manifestación del “ente" o "cosa" en la existencia, y de "correspondencia" entre el ente (realidad o cosa) y el ser (manifestación de la existencia del ente, realidad o cosa) mediante el lenguaje, es decir, los problemas del conocimiento se deben a que la relación sujeto-objeto de conocimiento no se puede establecer de manera esencial, sino, únicamente, a través del lenguaje como intermediario: se trata de una relación lógica. Con Aristóteles (1992, p. 8) es bueno admitir el concepto de ciencia, la categoría de teoría, la noción de primeros principios (Aristóteles, 1992, p. 75), y también es necesario reflexionar sobre la idea de primeras causas (Aristóteles, 1992, p. 76). De Schopenhauer (1997, pp. 215-216) se pueden destacar las enunciaciones de interpretación, explicación, existencia, sentimiento, intuición, concepción, abstracción, puntos de vista, etc. Con Bergson (1986, p. 7) se deben recuperar las ideas de ciencia, las nociones de posesión absoluta de la realidad, colocación del sujeto cognoscente en la realidad, tener la intuición de la realidad, aprehender la realidad fuera de toda expresión, traducción o representación simbólica, etc., así como las categorías de totalidad y evolución (Bergson, 1986, p. 33). En Natorp (1987, p. XIV) se pueden recuperar las ideas de conocimiento por principios y unidad del conocimiento; las nociones de inacabamiento de la ciencia y de la imposibilidad de conocer la esencia de la realidad (Natorp, 1987, p. XIV), así como el concepto de método (Natorp, 1987, p. XIV) y de totalidad (Natorp, 1987, p. 7). Con Ortega y Gasset (1998, p. 21) se puede afirmar que es muy afortunada la idea de filosofía, 
Revista Iberoamericana

de las Ciencias Sociales y

Humanísticas

ISSN: $2395-7972$

entendida como el pensar las ideas desde ciertas "suposiciones" o "convenciones"; también se debe considerar la idea de que los conceptos, ideas, categorías o nociones son solo teorías, hipótesis, suposiciones, presunciones, proposiciones o supuestos sobre la realidad, pues únicamente son espectros, visiones, apariciones o fantasmas de ella (Ortega y Gasset, 1998, p. 55).

\section{Justificación existencial, epistemológica, ontológica y lógica de la toma de partido sobre las "definiciones" de la categoría designada con la expresión filosofía}

Se considera que las "definiciones" de la idea expresada con la palabra filosofía elaboradas por todos y cada uno de los autores comentados - son pertinentes en términos existenciales, ontológicos y epistemológicos.

En Hessen (2009) se trata de "un intento del espíritu humano para llegar a una concepción del universo mediante la autorreflexión sobre sus funciones valorativas, teóricas y prácticas" (p. 9), los seres humanos reflexionamos sobre la realidad a partir de nuestras necesidades existenciales y, en este caso, se dice que la filosofía puede satisfacer necesidades de conocimiento de la realidad (función teórica), de toma de conciencia sobre las consecuencias de nuestros actos (función valorativa) y de toma de decisiones sobre nuestro comportamiento en el mundo (función práctica); la intención del sujeto cognoscente es la de determinar al objeto por conocer, es decir, conocer la realidad para tener conciencia de las consecuencias de sus actos sobre ella y, consecuentemente, poder tomar decisiones razonadas sobre su comportamiento en el mundo; por ello, es posible estimar que la "definición" de la idea expresada con el término filosofía que se comenta es de tipo "racional"; el alma humana tiene la intención de poner orden en la realidad para satisfacer sus necesidades de conocimiento, concientización y toma de decisiones.

Con Heidegger (2006) la entendemos como "un corresponder que traduce a lenguaje la llamada del ser del ente" (p. 66); todos los seres humanos sentimos y pensamos de manera diferente. Luego, entonces, debemos conformarnos con traducir a lenguaje la llamada del ser del ente, es decir, en el conocimiento no está presente la realidad en sí, sino solo un discurso sobre ella elaborado por el sujeto cognoscente. Esto permite establecer que la "definición" del concepto nombrado con la palabra filosofía - construido por Heidegger - también es 


\section{Revista Iberoamericana de las Ciencias Sociales y Humanísticas}

"racional" porque se avoca a la relación lógica entre el sujeto cognoscente y el objeto por conocer cuando establece que ella se reduce a lenguaje.

De Aristóteles (1992) se puede decir que la filosofía es "la ciencia teórica de los primeros principios y de las primeras causas", porque supone que la razón lee las impresiones de los sentidos utilizando, a manera de lupa, dichos principios:

El alma vegetativa y el alma animal constituyen tan solo en el hombre la materia de la realización de su propia forma: la razón (Nous-dianoeísthay). Por obra de esta se convierte, en efecto, el impulso (órexis) en voluntad (boúlesis) y la representación en conocimiento (episteme). Este añade, por así decirlo, como algo nuevo y más elevado a todas las actividades psíquicas que se derivan de la percepción (y de las que solamente participan los animales), pero solo puede realizarse en el hombre (p. 8).

Es posible estimar que la "definición" del concepto denominado con el término filosofía — construido por Aristóteles - es de tipo "intelectual" (leer en el interior). Se supone que los saberes tienen su origen primero en las percepciones de los sentidos y después en la "lectura" que el espíritu realiza de ellas utilizando para ello ciertos "principios" con los que pretende extraer la esencia de la realidad para realizar determinados fines. Para un intelectualista como Aristóteles "el conocimiento, en cuanto tal, es acto, y, al menos, ese acto es operación: a la operación corresponde un 'objeto"”, "la operación supone una facultad" (Polo, 2006, p. 15).

Para Schopenhauer (1997) la filosofía es un "interpretar y explicar lo existente, la esencia del mundo, que inconcreta, es decir, como sentimiento, se nos revela a cada uno de nosotros intuitivamente; presentarla en conceptos abstractos y precisos, y esto bajo todos los aspectos posibles y desde todos los puntos de vista" (p. 215), por lo que se estima que la definición del concepto expresado mediante el término filosofía -elaborado por Schopenhauer - es de tipo "sensual"; la realidad, a decir de este autor, se aprehende únicamente con los sentidos, de manera empírica. Son las experiencias vividas las que nos permiten conocer la esencia de los fenómenos.

En Bergson (1986) se define como "la ciencia que pretende prescindir de símbolos" (p. 7). Igual que Schopenhauer, la filosofía es entendida como una ciencia que no necesita de símbolos, sino de realidades, de experiencias vividas; es de apreciar que la definición del 


\section{Revista Iberoamericana \\ de las Ciencias Sociales y Humanísticas}

ISSN: $2395-7972$

concepto expresado con la voz filosofía para este autor es de tipo "sensual". La realidad no se conoce, se vive.

Con Natorp (1987) se entiende como "conocimiento por principios, mas no se dirige a los objetos sino a la unidad del mismo conocimiento" (p. XIV); es de tipo "racional", es decir, dicho discurso está elaborado a partir de la razón y el espíritu del autor pretende poner orden en el conocimiento por medio de principios, postulados, axiomas o máximas racionalistas.

En Ortega y Gasset (1998) se interpreta de este modo:

Toda idea es pensada y todo cuadro es pintado desde ciertas suposiciones o convenciones tan básicas, tan de clavo pasado para el que pensó la idea o pintó el cuadro, que ni siquiera repara en ellas y por lo mismo no las introduce en su idea ni en su cuadro, no las hallamos allí puestas sino precisamente supuestas y como dejadas a la espalda. Por eso, a veces, no entendemos una idea o un cuadro: nos falta la palabra enigma, la clave de la secreta convicción (p. 21).

Esto significa que se considera de tipo "racional”. Toda construcción de saberes, con conocimiento o no de quien los elabora, parte de determinadas suposiciones o convenciones que el sujeto cognoscente debiera conocer para entender mejor la realidad.

\section{Aclaración de la "definición” de la idea denominada con el término filosofía}

Si recuperamos algunas de las ideas principales de los autores comentados, es posible elaborar una definición que trate de abarcar todas las posturas analizadas, en los siguientes términos: la definición del término designado con la expresión filosofía se puede entender como una intensión del alma humana de explorar, describir, explicar, interpretar o comprender la realidad a partir de determinados principios que se deben suponer para resolver los grandes problemas del conocimiento, dependiendo de las facultades cognoscitivas que el sujeto cognoscente utilice para ello, a partir de sus necesidades existenciales, epistemológicas, ontológicas y lógicas en determinado momento, lugar y circunstancias. 
En donde:

- Por la palabra principios se debe entender los lógicos, ontológicos, epistemológicos y existenciales cuyo estudio será abordado en el siguiente apartado.

- Por la palabra supuestos se debe entender los términos, vocablos o voces con los que han sido designados los principios que indican movimiento y/o quietud (escepticismo, subjetivismo, relativismo, pragmatismo, dogmatismo y criticismo), orden y/o desorden (racionalismo, empirismo, intelectualismo y apriorismo), determinismo y/o indeterminismo (objetivismo, subjetivismo y dialéctica), existencia y/o inexistencia (realismo, idealismo y fenomenalismo), dualidad, unidad o diversidad (dualismo, monismo y pluralismo) que permiten construir un conocimiento de la realidad de tipo racional y/o sensual con conceptos y criterios de verdad inmanentes y/o trascendentes y con los que se intenta solucionar los grandes problemas del conocimiento.

- Por el enunciado problemas del conocimiento se debe entender las grandes incógnitas que a lo largo de la historia de la filosofía se han pretendido resolver desde diferentes supuestos, dependiendo de la facultad o facultades cognoscitivas que el sujeto cognoscente utilice para ello, sobre la posibilidad, el origen, la esencia, la clasificación y los conceptos y criterios de verdad del conocimiento, entre otros.

- Por lo expuesto como facultades cognoscitivas se debe imaginar a la razón y/o los sentidos que - más que esclarecer los grandes problemas del conocimientopermiten enderezar las necesidades existenciales, epistemológicas, ontológicas y lógicas.

- Por lo expresado como necesidades existenciales se deben concebir aquellas que tienen todo sujeto cognoscente de sobrevivir en este mundo, como son las de alimentación, vestido, habitación, educación, recreación, etc.

- Por la expresión necesidades epistemológicas se debe entender la necesidad de conocer la realidad que todo sujeto cognoscente tiene para estar consciente de sus alcances y limitaciones sobre ella y tomar la decisión más adecuada para resolver sus necesidades existenciales.

- Por lo expresado como necesidades ontológicas se deben entender los mismos principios, postulados, axiomas, supuestos, proposiciones o premisas que indiquen movimiento y/o quietud, orden y/o caos, determinismo y/o indeterminismo, existencia 
Revista Iberoamericana

de las Ciencias Sociales y

Humanísticas

ISSN: 2395 - 7972

y/o inexistencia, unidad, dualidad y/o pluralidad, racional y/o sensual, trascendencia y/o inmanencia.

- Por necesidades lógicas se comprenden todas aquellas que se refieren a la corrección del pensamiento.

\section{Justificación existencial, epistemológica, ontológica y lógica de la “definición” de la noción designada con la expresión filosofía}

La definición de la idea aludida con la expresión filosofía (entendida como una intensión del alma humana de explorar, describir, explicar, interpretar o comprender la realidad utilizando determinados principios que deben ser supuestos para resolver los grandes problemas del conocimiento, dependiendo de las facultades cognoscitivas que el sujeto cognoscente utilice para ello, a partir de necesidades existenciales, epistemológicas, ontológicas y lógicas) parece ser pertinente debido a que abarca cualquier intensión de conocimiento que tenga cualquier sujeto cognoscente de conocer cualquier realidad.

\section{Análisis y síntesis de la definición de la categoría nombrada con la palabra filosofía}

Si se "descompone" la "definición" de la idea que se pretende expresar con la palabra filosofía en las partes que la forman, se tiene lo siguiente:

- Los seres humanos tienen necesidades de alimentación, vivienda, vestido, recreación, educación, realización y muchas más para sobrevivir en este mundo. A dichas carencias se les puede denominar necesidades existenciales.

- Para satisfacer sus necesidades existenciales los seres humanos necesitan conocer la realidad. Estas insuficiencias se pueden designar como necesidades epistemológicas.

- Para conocer la realidad los seres humanos tienen la necesidad de partir de determinados principios del conocimiento o de la ciencia. A estas privaciones se les puede distinguir como necesidades ontológicas.

- Finalmente, las necesidades ontológicas se relacionan con otras carencias lógicas; es decir, las primeras se refieren al ente, las segundas a la manifestación de este en la experiencia por medio del lenguaje. A este último tipo de necesidades se les puede designar como necesidades lógicas. 
- De igual manera, para satisfacer sus necesidades existenciales los seres humanos cuentan con dos facultades cognoscitivas: su razón y sus sentidos.

- La razón y/o los sentidos son utilizados por los seres humanos como "facultades cognoscitivas" (función teórica), como facultades de toma de conciencia sobre las consecuencias de sus actos (función valorativa) y como facultades de toma de decisiones sobre su comportamiento en el mundo (función práctica).

- También sucede que, para resolver sus problemas existenciales los seres humanos necesitan conocer la realidad (función teórica) y tener conciencia de las consecuencias de sus actos (función valorativa) para tomar decisiones sobre su comportamiento en el mundo (función práctica).

- Para conocer la realidad los seres humanos se tienen que enfrentar a los problemas del conocimiento en virtud de que se ha demostrado que la realidad no se puede conocer en esencia. Se pueden mencionar algunas grandes incógnitas a las que no se les ha podido dar una solución definitiva: la posibilidad, origen, esencia, clasificación y concepto, y criterio de verdad del conocimiento, entre otros.

- Los problemas del conocimiento mencionados se han tratado de resolver de cuatro modos diferentes, dependiendo de la o las facultades cognoscitivas que se utilicen para ello: la razón y/o los sentidos. Dichas soluciones no son esenciales, solo se suponen.

- Los problemas del conocimiento se pretenden resolver con determinados principios que deben ser supuestos y que se derivan de la o las facultades cognoscitivas que se utilicen para ello.

- Todos y cada uno de los principios se relacionan con todos y cada uno de los cinco principales problemas del conocimiento humano.

- Todos y cada uno de los principios establecen uno y solo un supuesto con el que se pretende resolver los problemas filosóficos del conocimiento humano, dependiendo de la facultad cognoscitiva que se utilice para ello.

Si se vuelven a juntar las partes que componen la definición del concepto denominado con la palabra filosofía en un todo, se tiene lo siguiente: la filosofía es el propósito del espíritu humano de entender la realidad a partir de determinados supuestos que se derivan de ciertos principios nacidos de las facultades cognoscitivas del sujeto cognoscente, pretendiendo resolver con ellos los problemas del conocimiento, dependiendo de sus necesidades 
Revista Iberoamericana

de las Ciencias Sociales y

Humanísticas

ISSN: $2395-7972$

existenciales, epistemológicas, ontológicas y lógicas en determinado momento, lugar y circunstancias.

\section{El conductismo. Un mapeo del concepto}

\section{Necesidades existenciales, epistemológicas, ontológicas y lógicas del sujeto cognoscente en la teoría conductista}

Ya se comentó que los seres humanos tienen necesidades de alimentación, vivienda, vestido, recreación, educación, realización y muchas más para sobrevivir en este mundo. A dichas carencias se les pueden denominar necesidades existenciales. Al respecto, Skinner (1994) se pregunta:

¿Es realmente posible la ciencia?, ¿puede explicar cualquier aspecto del comportamiento humano?, ¿qué métodos puede emplear?, ¿sus leyes son tan válidas como las de la física y la biología?, ¿conducirá hacia una tecnología y, en tal caso, qué papel desempeñará en los asuntos humanos? (...) ¿hasta qué punto lo que se ha dicho vale algo? (p. 7).

Ante estos cuestionamientos es posible apreciar que se plantea la necesidad epistemológica de cómo explicar el comportamiento debido a una necesidad existencial de resolver los asuntos humanos. Dicha necesidad existencial va más allá de la mera explicación del comportamiento humano (necesidad epistemológica); en un nivel superior, de lo que se trata es de poderlo predecir (otra necesidad epistemológica) para poder controlarlo (otra necesidad existencial): “¿Por qué la gente se comporta de la forma como lo hace? (...) ¿cómo podría una persona anticipar y, por tanto, prepararse para lo que otra persona iba a hacer? (...) ¿cómo se podría inducir a una persona a comportarse de cierta manera?” (Skinner, 1994, p. 12). Para resolver sus problemas existenciales los seres humanos necesitan conocer la realidad (función teórica del espíritu) y tener conciencia de las consecuencias de sus actos (función valorativa de la razón) para tomar decisiones sobre su comportamiento en el mundo (función práctica de la voluntad). Según Skinner (1994), "si queremos predecir lo que hará una persona, ¿cómo podemos descubrir las causas mentales de su comportamiento? y ¿cómo podemos producir los sentimientos y estados de la mente que la inducirán a comportarse de una manera determinada?” (p. 13). La idea es conocer el comportamiento humano para predecirlo; predecirlo para controlarlo, y controlarlo para resolver los asuntos humanos relacionados con la conducta. 


\section{Principios del conocimiento a partir de los cuales el sujeto cognoscente pretende hacer ciencia de la conducta}

Buscando comprender los principios de la ciencia, en general, se encontró esta definición del concepto de filosofía de Aristóteles (1992): "Ciencia teórica de los primeros principios y de las primeras causas" (p. 8). Siguiendo a Aristóteles (1992), el concepto expresado con la voz primeros principios se entiende, en un primer momento, como el "punto de partida de la cosa" (p. 75); en un segundo momento, como "aquello mediante lo que puede hacerse mejor una cosa; por ejemplo, el principio de una ciencia” (p. 75), y, en un tercer momento, como "la parte esencial y primera de donde proviene una cosa" (p. 75).

Se supone que los primeros principios son válidos para cualquier ciencia: "Un 'principio' es una proposición verdadera, absolutamente evidente, universal y necesaria. Por tanto, no necesita demostración, sino que, por el contrario, está supuesta en cualquier demostración" (Gutiérrez, 2005, p. 157).

Santo Tomás de Aquino, siguiendo a Aristóteles, propone tres características esenciales de todo primer principio:

Condiciones que debe realizar el principio más cierto... 1..$^{\circ}$ Debe ser un principio sobre el cual sea imposible todo error, pues uno se engaña sobre las cosas que no conoce; $2 .^{\circ}$ No debe suponer ninguna verdad anterior, pues es necesario para conocer cualquier cosa; $3 .^{\circ}$ Debe estar en nosotros naturalmente, con anterioridad a toda investigación del espíritu, puesto que esta investigación lo supone (Garrigou-Lagrange, 1980, p. 203).

Todo principio se refiere al ser y a lo que se predica del ser:

El análisis de nuestras ideas muestra de un modo indiscutible que la idea enteramente primera implícita en todas las demás es la idea de ser; de ello se deduce legítimamente que el juicio enteramente primero debe tener por sujeto el ser y por predicado lo que primeramente conviene al ser (GarrigouLagrange, 1980, p. 203).

¿Qué es aquello que primeramente conviene al ser?, ¿qué es lo que encaja primeramente con la ciencia? Los primeros principios, o sea, aquello que es conveniente primeramente a todos ser, pueden ser lógicos (discursivos), ontológicos (acción, pasión o estado en que se encuentra el ser del ente en determinado lugar, tiempo y circunstancias), epistemológicos (teorías del conocimiento o de la ciencia) y existenciales. Es decir, se supone 


\section{Revista Iberoamericana de las Ciencias Sociales y Humanísticas}

que el ser del ente se manifiesta en la existencia por medio del lenguaje. Para aceptar esto es necesario recurrir a la definición del concepto expresado con el término filosofía elaborado por Heidegger (2006) como "un corresponder que traduce a lenguaje la llamada del ser del ente" (p. 66).

Si por la expresión ente se entiende todo ser, cosa u objeto que tiene una existencia propia e independiente en la realidad, entonces la enunciación de la categoría nombrada con la expresión filosofía por Heidegger se puede interpretar como la correspondencia que se puede establecer entre el ente (como posibilidad de existencia) y el ser (como experiencia) a través del lenguaje; es decir, como la manifestación del ser del ente mediante expresiones, frases, palabras, locuciones o voces; o sea, que cualquier ente o cosa se manifiesta en la existencia por medio de la comunicación mediante el lenguaje.

Algunos primeros principios lógicos, ontológicos, epistemológicos y existenciales que se supone que "convienen" a toda ciencia o conocimiento, son los siguientes:

- Principio lógico de no contradicción: "Es imposible afirmar y negar un mismo predicado a un mismo sujeto al mismo tiempo y bajo el mismo aspecto" (Gutiérrez, 2005, p. 158). También se puede formular así: "Dos proposiciones contradictorias no pueden ser a la vez verdaderas" (Gutiérrez, 2005, p. 158) y sus correspondientes principio ontológico de quietud:

Solo el ser es, el no-ser no puede ser (...), el ser es igual a la materia, al espacio lleno, el no-ser es el espacio vacío. Por tanto, no puede haber ningún espacio vacío y, de consiguiente, tampoco movimiento, puesto que este significa que las cosas materiales cambian de lugar a través del espacio vacío; y como el ser pleno se encuentra por todas partes, no puede haber ningún cambio; la materia sigue siendo materia en todas las circunstancias, y todo devenir y pasar es apariencia engañosa (Simmel, 1946, pp. 56-58).

Y epistemológico y existencial de predicción. A decir de Skinner (1994):

Hay una clase de predicción posible sobre el <<principio〉> de que es probable que la gente haga de nuevo lo que ha hecho frecuentemente; las personas siguen las costumbres porque es habitual hacerlo... El descubrimiento de $<<$ principios $>>$ de organización en la estructura del comportamiento... pueden hacer posible la predicción de casos de comportamiento que no han ocurrido previamente (p. 14). 


\section{Revista Iberoamericana \\ de las Ciencias Sociales y Humanísticas}

ISSN: $2395-7972$

Ello permite suponer que para conocer el comportamiento humano y poder predecirlo para controlarlo es preciso partir de determinados principios del conocimiento; en este caso, el principio de que "es probable que la gente haga de nuevo lo que ha hecho frecuentemente", es decir, las costumbres o los hábitos que se derivan de otros principios superiores de orden lógico y que se puede expresar como "es imposible afirmar y negar un mismo predicado a un mismo sujeto al mismo tiempo y bajo el mismo aspecto" o como "dos proposiciones contradictorias no pueden ser a la vez verdaderas" que, a su vez, se relacionan con principios ontológicos de reposo o quietud.

Para el conductismo "la educación es uno de los procedimientos que emplea la sociedad para 'controlar' la conducta de las personas” (Guzmán y Hernández Rojas, 1993, p. 14). Posiblemente lo anterior se debe a que se supone que los objetivos conductuales no se mueven, es decir, que permanecen siempre quietos porque para establecer los juicios sobre los fines de la educación, en este caso, el conductista parte de principios lógicos de no contradicción, ontológicos de reposo o quietud y epistemológicos y existenciales de predicción. En ese entendido, es posible presumir que la conducta humana se puede conocer, predecir y controlar.

- Principio lógico de identidad: "A es necesariamente A" (Gutiérrez, 2005, p. 159) y su correspondiente principio ontológico de determinismo: "El ser es; el no ser no es" (Gutiérrez, 2005, p. 159). "El mismo ser no puede ser y no ser al mismo tiempo y bajo la misma relación” (Garrigou-Lagranje, 1980, p. 204). No sería correcto afirmar que una cosa es y, a la vez y bajo el mismo aspecto, negar que lo sea. Es otra forma de enunciar el principio de no contradicción. "Aristóteles dice, desde el punto de vista lógico: 'es imposible que el mismo atributo pertenezca y no pertenezca al mismo sujeto, al mismo tiempo y bajo la misma relación"” (Garrigou-Lagrange, 1980, p. 204). Lo que primeramente conviene al ser (sujeto) es que las razones en que se apoyan los juicios sobre su sustancia y sus accidentes (predicado) únicamente le pertenezcan y, no que le pertenezcan y, al mismo tiempo y bajo la misma relación, no le pertenezcan.

En el conductismo, estos dos principios nos permiten arribar a un tercero: el principio epistemológico y existencial de poder tratar a los alumnos como objetos del acto educativo. En esta teoría psicológica aplicada a la educación, la definición de la categoría expresada con la palabra alumno se entiende como "el objeto del acto educativo, en cuanto receptor de todo 


\section{Revista Iberoamericana de las Ciencias Sociales y Humanísticas}

ISSN: $2395-7972$

el proceso instruccional diseñado por el maestro" (Guzmán y Hernández Rojas, 1993, p. 16). Es decir, después de ser sometidos a procesos de aprendizaje (objetos de estudio), los alumnos (sujetos cognoscentes) quedarán determinados o indeterminados por los contenidos; es decir, aprobados o reprobados.

Es probable que lo anterior sucede porque el conductista estima que el sujeto cognoscente (alumno) puede ser determinado por el objeto de conocimiento (los contenidos de la enseñanza) debido a que, para elaborar los juicios sobre la definición del concepto expresado con el término alumno, el conductista parte de principios lógicos de identidad, ontológicos de determinismo y epistemológicos y existenciales de pensar al alumno como objeto del acto educativo, cuya conducta puede ser modificada por los contenidos conductuales.

- Principio lógico de tercero excluido: "No hay medio entre dos proposiciones contradictorias" (Gutiérrez, 2005, p. 159) y sus correspondientes principios ontológicos: "Cualquier cosa es o no es, no cabe término medio" (Gutiérrez, 2005, p. 159) y de exclusión, y epistemológico y existencial de control. Lo que primeramente conviene al ser, ente, cosa o sujeto es que lo que se predique sobre el mismo sea verdadero o falso (exclusión), pero no las dos cualidades al mismo tiempo y bajo la misma relación.

Para el conductismo, la evaluación “juega un papel crucial para mejorar la enseñanza, ya que el estarla checando continuamente permite detectar en forma expedita sus aciertos y errores. Es imprescindible utilizar instrumentos objetivos para constatar el logro de los objetivos conductuales" (Guzmán y Hernández Rojas, 1993, p. 18). Es decir, se trata de excluir (por medio de exámenes de objetivos conductuales) y descartar, o sea, separar, a los alumnos que no se dejen determinar por los objetivos conductuales, o sea, de controlarlos.

Posiblemente lo anterior se debe a que se supone que la conducta de los alumnos se puede conocer y, por lo mismo, predecir y controlar porque para elaborar la definición del concepto expresado con la voz evaluación el conductista parte de principios lógicos de tercero excluido, ontológicos de exclusión y epistemológicos y existenciales de control de la conducta humana.

Los dos principios que siguen solo se refieren a lo ontológico. En términos lógicos, coinciden en todo con los principios mencionados anteriormente. 


\section{Revista lberoamericana de las Ciencias Sociales y Humanísticas}

- Principio ontológico de razón suficiente: "Todo ente tiene una razón suficiente de su existencia. O bien: Todo ser tiene una razón suficiente de ser" (Gutiérrez, 2005, p. 159). También se puede interpretar en términos de orden. Si se considera cuidadosamente este primer principio ontológico, se advertirá que se refiere al ser. Todo fenómeno tiene necesidad de una explicación, de una razón de ser:

Todo lo que es tiene su razón de ser, y consiguientemente todo es inteligible; y no: todo es inteligible, consiguientemente todo debe tener para nosotros una razón de ser. Así como la inteligencia conoce el ser antes de conocerse a sí misma y no es inteligible por sí misma sino en función del ser (como una viviente relación al ser), mientras que el ser es inteligible por sí; así también ella descubre los primeros principios en el ser, como leyes del ser, antes de descubrirlos como leyes del pensamiento; y estos principios no son leyes del pensamiento (esencialmente relativo al ser), sino porque son primeramente leyes del ser (Garrigou-Lagrange, 1980, pp. 217-218).

Y su correspondiente principio epistemológico y existencial de resolver los problemas humanos relacionados con la educación, en relación con la teoría conductista del aprendizaje. Se trata, como ya se ha establecido, de conocer la conducta humana para poder predecirla y controlarla. "Para los conductistas aprender es una modificación relativamente permanente del comportamiento observable de los organismos como fruto de la experiencia" (Guzmán y Hernández Rojas, 1993, p. 15). Es la razón humana la que tiene la necesidad de poner orden en la conducta de los alumnos con motivo de resolver los problemas existenciales de la educación.

Es probable que lo anterior sucede porque los conductistas estiman que la conducta humana es modificable debido a que para elaborar la definición de la idea mentada con la expresión aprendizaje se apoya en principios ontológicos racionales, es decir, el alma humana trata de poner orden en el aprendizaje y epistemológicos y existenciales de solución de la problemática del aprendizaje.

El principio ontológico de razón suficiente es análogo al principio ontológicoracional de causalidad:

- Principio ontológico de causalidad: "Todo lo que existe tiene una causa de su existencia (...) aun cuando se nieguen las causas, de todas maneras, subsiste la necesidad de una razón para explicar el ser de las cosas" (Gutiérrez, 2005, p. 160) y 
Revista Iberoamericana

de las Ciencias Sociales y

Humanísticas

ISSN: $2395-7972$

su correspondiente principio epistemológico y existencial de dirección y control del proceso de aprendizaje. De acuerdo con la lógica deductiva, que es la lógica de todo racionalista, existen dos modos que convienen directamente al ser de los entes, uno positivo y otro negativo. El modo positivo consiste en afirmar que el ser de los entes está determinado, su naturaleza determinada lo constituye propiamente, es decir, le es conveniente:

Encontramos dos modos que convienen a todo ser en sí considerado: uno positivo y otro negativo (Garrigou-Lagrange, 1980, p. 206).

El modo positivo consiste en que cada ser es una cosa determinada... El juicio supremo que debe afirmar lo que primeramente conviene al ser tiene, pues, como fórmula: "Todo ser es una cosa determinada, una naturaleza determinada que propiamente lo constituye". Es de este modo que se dice sin tautología: Dios es Dios... A es A. Si se da a la proposición por sujeto el ser, en el predicado hay que expresar la naturaleza del ser, lo que lo define, y decir no "el ser es el ser" sino "el ser es lo que es o puede ser". Y como es manifiesto que este primer predicado conviene al primer sujeto por sí mismo, se puede precisar la formula y decir: "Todo ser es y es por sí mismo una naturaleza determinada que propiamente lo constituye". Esta fórmula no es más una tautología; hasta hay una filosofía que niega su verdad: la filosofía del devenir, cautivada por las apariencias sensibles, que niega que haya cosas para no admitir sino acciones, que define lo real no por lo que es, sino por lo que se hace y cambia sin cesar, que rehúsa por consiguiente ver distinciones reales (Garrigou-Lagrange, 1980, p. 207).

El modo negativo que se supone que conviene a todo ser en sí considerado, es decir, a todo ser del ente, es la unidad:

En cuanto al modo negativo que conviene a todo ser en sí considerado, es la unidad, que se opone a la multiplicidad, como la identidad que se opone a la diversidad... Si todo ser es una determinada naturaleza, que propiamente lo constituye, síguese que como tal no está dividido; si estuviese dividido sería y no sería bajo el mismo aspecto lo que propiamente lo constituye. Si es simple, no solo es individuo sino indivisible; si es compuesto, deja de ser cuando es dividido... La unidad, siendo una propiedad del ser, varía con él. En efecto, el 
Revista Iberoamericana

de las Ciencias Sociales y

Humanísticas

ISSN: $2395-7972$

ser se dice primeramente de la substancia, después de la cantidad, de la cualidad, etc., de los diferentes accidentes que son por cierto algo real; del mismo modo la unidad tiene las correspondientes múltiples acepciones: la identidad es la unidad de esencia o de substancia, la igualdad es la unidad de cantidad, la semejanza es la unidad de cualidad. Lo que expresa pues el principio "todo ser es uno y el mismo", es la identidad funcional de todo ser consigo mismo (Garrigou-Lagrange, 1980, p. 208).

Se supone que la unidad se opone a la multiplicidad, la identidad a la diversidad, el determinismo al indeterminismo, la indivisibilidad a la divisibilidad, el todo a las partes, lo simple a lo complejo, lo compuesto a lo dividido, etc. En la teoría conductista se privilegia el control y la dirección del proceso de aprendizaje en el profesor: "Esta postura asigna al profesor un papel directivo y controlador del proceso de aprendizaje; se le define como 'ingeniero conductual' que moldea comportamientos positivamente valorados por la escuela" (Guzmán y Hernández Rojas, 1993, p. 16).

Posiblemente lo anterior se debe a que se supone que la conducta de los alumnos se puede conocer y, por lo mismo, predecir y controlar porque para elaborar la definición del enunciado expresado con el enunciado el papel del maestro el conductista parte de principios lógicos de no contradicción, identidad, tercero excluido, razón suficiente y causalidad; de principios ontológicos de quietud, orden, determinismo, dualismo, etc.; y de principios epistemológicos y existenciales de dirección y control del proceso de aprendizaje.

\section{Conductismo y filosofía. Una relación lógica, ontológica, epistemológica y existencial}

Para efectos de establecer las relaciones de carácter lógico, ontológico, epistemológico y existencial entre la filosofía y la teoría psicológica del conductismo, es pertinente considerar la definición del concepto expresado con el término filosofía, elaborado en el apartado primero de este trabajo como el propósito del espíritu humano de entender la realidad a partir de determinados supuestos que se derivan de ciertos principios nacidos de las facultades cognoscitivas del sujeto cognoscente, pretendiendo resolver con ellos los problemas del conocimiento, dependiendo de sus necesidades existenciales, epistemológicas, ontológicas y lógicas en determinado momento, lugar y circunstancias. 
Revista Iberoamericana

de las Ciencias Sociales y

Humanísticas

ISSN: $2395-7972$

La filosofía proporciona los supuestos para resolver los problemas del conocimiento. Los supuestos dependen de las facultades cognoscitivas que utilice el sujeto cognoscente para resolver los problemas del conocimiento. Las facultades cognoscitivas con las que se pretendan resolver los problemas del conocimiento mediante determinados supuestos dependerán de las necesidades del sujeto cognoscente en determinado tiempo, lugar y circunstancias, lo que le orillará a utilizar determinados principios de orden lógico, ontológico, epistemológico y existencial en la construcción de los discursos conductistas.

\section{¿Cuáles son los principales problemas del conocimiento de la conducta humana?}

Ya se observó, en la segunda parte de este trabajo, que Skinner (1994) precisa los problemas del conocimiento de la conducta humana de manera muy esclarecedora:

¿Es realmente posible la ciencia? ¿Puede explicar cualquier aspecto del comportamiento humano? ¿Qué métodos puede emplear? ¿Sus leyes son tan válidas como las de la física y la biología? ¿Conducirá hacia una tecnología y, en tal caso, qué papel desempeñará en los asuntos humanos? ... ¿Hasta qué punto lo que se ha dicho vale algo? (p. 7).

Si se traducen —en términos de teoría de la ciencia— los problemas mencionados, se puede establecer que el autor citado se refiere a la posibilidad, origen, esencia, composición y conceptos, y criterios de verdad del conocimiento humano, en términos de Hessen (2009).

En la tabla 1 de este estudio se muestran los principales problemas que, al igual que cualquier otra teoría psicológica o sociológica del comportamiento humano, el conductismo pretende resolver: 
Revista Iberoamericana

de las Ciencias Sociales y

Humanísticas

ISSN: $2395-7972$

Tabla 1. Los problemas filosóficos del conocimiento de la conducta humana

\begin{tabular}{|c|c|c|}
\hline $\mathrm{N}^{\circ}$ & PREGUNTA & TEMA \\
\hline 1 & ¿Es posible conocer la conducta humana? & $\begin{array}{l}\text { El problema de la } \\
\text { posibilidad del } \\
\text { conocimiento de la } \\
\text { conducta humana }\end{array}$ \\
\hline 2 & $\begin{array}{l}\text { ¿Cuál es la fuente del conocimiento de la conducta } \\
\text { humana? }\end{array}$ & $\begin{array}{l}\text { El problema del } \\
\text { origen del } \\
\text { conocimiento de la } \\
\text { conducta humana }\end{array}$ \\
\hline 3 & $\begin{array}{c}\text { ¿Cuál es la esencia del conocimiento de la conducta } \\
\text { humana? }\end{array}$ & \multirow{4}{*}{$\begin{array}{l}\text { El problema de la } \\
\text { esencia del } \\
\text { conocimiento de la } \\
\text { conducta humana }\end{array}$} \\
\hline 3.1 & $\begin{array}{l}\text { El problema de la relación de conocimiento: ¿quién } \\
\text { determina a quién en una relación de conocimiento de la } \\
\text { conducta humana: ¿el sujeto al objeto, el objeto al sujeto o } \\
\text { ambos se determinan recíprocamente? }\end{array}$ & \\
\hline 3.2 & $\begin{array}{l}\text { El problema de la existencia de la conducta humana: } \\
\text { ¿puede existir la conducta humana con independencia del } \\
\text { sujeto cognoscente? }\end{array}$ & \\
\hline 3.3 & $\begin{array}{l}\text { El problema de la composición de la conducta humana: ¿la } \\
\text { conducta humana es única, dual o múltiple? }\end{array}$ & \\
\hline 4 & ¿Cómo se tipifica la conducta humana? & $\begin{array}{l}\text { El problema de la } \\
\text { clasificación de la } \\
\text { conducta humana }\end{array}$ \\
\hline 5 & $\begin{array}{l}\text { ¿Cuáles son los criterios y conceptos de verdad que nos } \\
\text { permiten aceptar un conocimiento de la conducta humana } \\
\text { como verdadero o rechazarlo por falso? }\end{array}$ & $\begin{array}{l}\text { El problema de la } \\
\text { verdad del } \\
\text { conocimiento de la } \\
\text { conducta humana }\end{array}$ \\
\hline
\end{tabular}

Fuente: Elaboración propia 


\section{¿Con qué facultad o facultades cognoscitivas se pretenden resolver los problemas del conocimiento de la conducta humana?}

Cuando Skinner (1986) dice que "al intentar resolver los problemas que nos afectan en nuestro mundo actual, espontáneamente echamos mano de aquello que somos capaces de hacer mejor. Buscamos seguridad, y nuestra seguridad es la ciencia y la tecnología” (p. 5), es posible observar que pretende resolver el problema del origen del conocimiento de manera racional, es decir, utilizando únicamente como facultad cognoscitiva su razón y, por lo mismo, supone que existe un orden o, posiblemente, lo está buscando, debidamente establecido, que se manifiesta en la ciencia y la tecnología y que le dará seguridad para resolver los problemas conductuales de la humanidad. Es el alma o razón del autor citado en busca de orden para asegurar la solución de los problemas de conducta que afectan al mundo, mediante la ciencia y la tecnología establecidas, que le darán la seguridad para conocer, predecir y controlar la conducta humana.

Es así que Skinner (1986) se expresa de la siguiente manera sobre la forma de solucionar los problemas conductuales mundiales: "Lo que necesitamos es una tecnología de la conducta (...). Podríamos solucionar nuestros problemas con la rapidez suficiente si pudiéramos ajustar, por ejemplo, el crecimiento de la población mundial con la misma exactitud con que determinamos el curso de una aeronave" (p. 5). Es decir, el problema de la relación sujeto-objeto de conocimiento se pretende resolver con la razón, de tal manera que el objeto de conocimiento (la tecnología de la conducta humana) determine al sujeto cognoscente (la humanidad) en el entendido de que ella se aplique a modificar sus conductas de conformidad con dicha tecnología.

La tecnología de la conducta se dirige a todos los humanos para convencerlos de que -mediante reforzadores condicionados o de cualquier otro tipo- ajusten su comportamiento a determinadas pautas, pero si algunos no quedan convencidos, la razón o inteligencia de Skinner dividirá la realidad en dos polos opuestos e irreconciliables entre sí (presteza-renuencia, castigos-premios, desarrollo-subdesarrollo) para justificar la exclusión de ellos de manera racional: 


\section{Revista Iberoamericana \\ de las Ciencias Sociales y Humanísticas}

ISSN: $2395-7972$

El niño que necesita verse espoleado y regañado es algo menos que un ser humano plenamente desarrollado. Ansiamos verlo acudir con presteza a sus obligaciones no porque cada paso que se produzca sea en respuesta a las amonestaciones verbales de su madre, sino porque determinadas contingencias temporales, entre las cuales se ha castigado la renuencia y se ha reforzado la presteza, han actuado para realizar un cambio en su conducta. Llámesele a esto mejor organización, más sensibilidad o la realidad o como se quiera. El hecho escueto es que el niño ha pasado de un control verbal temporal ejercido por sus padres a ser controlado por ciertas facetas inexorables del medio (Skinner, 1985, p. 240).

Ello se debe a que el autor comentado pretende resolver el problema de la composición de la realidad de manera racional. A pesar de que se propone toda una tecnología para modificar la conducta de los seres humanos a partir de determinadas pautas y con ciertos reforzadores condicionados o no, lo cierto es que todo ello lo está inventando (y no descubriendo, como se pretende hacer ver) la razón o espíritu del autor comentado; es decir, el problema de la existencia de la realidad se pretende resolver de manera racional con ideas que nada tienen que ver con la realidad, es decir, inventadas por la inteligencia del autor:

Aunque no sea el terapeuta quien elija, aunque recomiende la “autorrealización", el control que ejerce no ha cesado y se mantiene dispuesto a intervenir tan pronto como lo exigen las circunstancias, cuando, por ejemplo, el cliente opta por perfeccionarse aún más en el arte de la mentira o cuando decide asesinar a su jefe (Skinner, 1985, p. 239).

Se supone que si ese control de origen racional o mental determina a la humanidad a comportarse como lo establece el conductismo, los problemas conductuales de la humanidad estarán resueltos y que ello puede ser posible mediante el uso de tecnologías de la conducta; una de ellas sería, por ejemplo, la admiración al comportamiento del otro: "La práctica de la admiración representa una parte importante de la cultura; porque la conducta, que de otro modo se debilitaría, gracias a la ayuda de aquella se fija y se mantiene" (Skinner, 1985, p. 229). Ello se debe a que el autor comentado pretende resolver el problema de la posibilidad del conocimiento de manera racional, suponiendo que es posible conocer, predecir y 


\section{Revista Iberoamericana \\ de las Ciencias Sociales y Humanísticas}

ISSN: $2395-7972$

controlar la conducta humana, en virtud de que estima, de igual modo, que la realidad conductual no cambia, mientras se refuerce:

Toda enumeración de valores es una enumeración de reforzadores, ya sean condicionados o de otro tipo. Estamos constituidos de forma tal que, en determinadas circunstancias, el alimento, el agua, el contacto sexual y otras cosas harán más probable que cualquiera que sea la conducta que las origina vuelva a producirse (...). Un organismo puede verse reforzado —inducido a “elegir”- casi por cualquier cosa que sea (Skinner, 1985, p. 238).

A manera de cierre, con conocimiento o no del hecho, el conductista pretende conocer la conducta humana utilizando como facultad cognoscitiva su alma o razón pretendiendo resolver con ella los principales problemas filosóficos del conocimiento (posibilidad, origen, esencia — relación sujeto-objeto-, existencia y composición de la realidad) debido a la necesidad existencial de predecirla y controlarla; es decir, de poner orden en el comportamiento humano.

\section{¿Cuáles son los supuestos filosóficos con los que el sujeto cognoscente de la teoría conductista pretende resolver los problemas del conocimiento?}

Una consecuencia necesaria de pretender conocer el comportamiento de la humanidad resolviendo los problemas fillosóficos del conocimiento con la razón, como ya se observó en el apartado anterior, es que el conductista se ubica, también con conocimiento o no del hecho, en supuestos filosóficos racionales. Cuando Skinner (1985) dice: "Si valoramos las conquistas y objetivos de la democracia, no debemos negarnos a aplicar la ciencia a la planificación y construcción de patrones culturales, pese a que nos encontremos entonces en cierto sentido también en la posición de controladores" (p. 241), se puede interpretar en el sentido de que la finalidad de controlar la conducta humana, es sinónimo de inspeccionarla, vigilarla, intervenirla, registrarla y fiscalizarla; es decir, determinar a la humanidad para que se comporte de acuerdo con determinados modelos, esquemas, pautas, estándares o moldes culturales.

En ese entendido, es posible estimar que se pretende conocer, predecir y controlar el comportamiento humano intentando resolver el problema de la relación sujeto-objeto de conocimiento de manera objetiva (se supone que, en una relación de conocimiento, el objeto 


\section{Revista Iberoamericana \\ de las Ciencias Sociales y Humanísticas}

ISSN: 2395 - 7972

determina al sujeto), debido a que la facultad cognoscitiva que se está empleando para ello es la razón o alma humana.

Se estima que, si ese control de origen racional o espiritual no es aceptado por todos los seres humanos a los que va dirigido, el alma del autor comentado dividirá la conducta en dos para justificar la exclusión de aquellos que no se dejen determinar por el orden establecido. Así se puede decir con el autor comentado lo siguiente:

Los individuos que conviven dentro de unos determinados grupos ejercen un control mutuo de acuerdo con una técnica que no sin razón adopta el nombre de "ética". Cuando un individuo se comporta de manera aceptable a ojos del grupo, recibe la admiración, la aprobación, el afecto y otros muchos reforzamientos que aumentan la probabilidad de que dicho individuo siga comportándose del mismo modo. Cuando su conducta no es aceptable, se ve criticado, censurado, zaherido o, en ciertos casos, castigado. En el primer caso el grupo califica al individuo de "bueno"; en el segundo, de "malo". Esta práctica se encuentra tan profundamente arraigada en nuestra cultura que a menudo no admitimos que se trata de una técnica de control. Y, sin embargo, casi siempre estamos implicados en dicho control, pese a que tanto los reforzamientos como los castigos impuestos acostumbren a ser sutiles (Skinner, 1985, p. 229).

Se puede apreciar que ello se debe a que el autor comentado pretende conocer, predecir y controlar el comportamiento humano, intentando resolver el problema de la composición de la realidad de manera racional o intelectual, y ubicándose por lo mismo en el supuesto filosófico que Hessen (2009) llama dualismo, porque se estima o supone que la conducta humana se encuentra dividida en dos polos opuestos, contradictorios e irreconciliables entre sí: la buena y la mala conducta, la conducta admirable y la conducta reprochable etc. En ese mismo tenor dice lo siguiente:

La ciencia está aumentando drásticamente nuestra capacidad de influir, cambiar, moldear - en una palabra - controlar la conducta humana (...). Ha identificado también ciertas condiciones o variables que pueden utilizarse para predecir y controlar la conducta según una tecnología nueva y cada vez más rigurosa (...). El estudio experimental de la conduta nos lleva más allá de complejos o inaccesibles "principios", "factores", etcétera, hasta unas 


\section{Revista Iberoamericana \\ de las Ciencias Sociales y Humanísticas}

ISSN: $2395-7972$

variables susceptibles de ser directamente manipuladas (Skinner, 1985, p. 227).

La cita anterior se puede interpretar en el sentido de que la mente o razón del autor comentado pretende poner orden en la conducta de los seres humanos, buscando razones para convencer a su público de que es posible conocerla, predecirla y controlarla mediante el establecimiento de determinados escenarios, ambientes, situaciones, circunstancias o contextos. Ello se debe a que, con conocimiento o no del hecho, se intenta conocer el comportamiento humano, pretendiendo resolver el problema del origen del conocimiento de manera racional o mental, suponiendo que en la naturaleza humana existe un orden al que puede y debe ajustarse la conducta de los demás. A este supuesto filosófico Hessen (2009) le llama racionalismo porque se origina en la razón o alma del sujeto cognoscente.

Una vez que con el uso de la tecnología de la conducta se logra convencer a la mayoría de los seres humanos para que ajusten su comportamiento a determinados patrones culturales propuestos de manera racional o espiritual, las personas se adaptan, conforman, acomodan, acondicionan o se aclimatan a ellos:

Las personas se comportan de manera, decimos, que se amoldan a unas pautas éticas, políticas o religiosas, porque están reforzadas para proceder de este modo. La conducta resultante puede tener consecuencias de gran alcance para la supervivencia de la pauta a la que aquella se conforma. Y, nos guste o no, la supervivencia constituye el criterio último (Skinner, 1985, p. 238).

Es decir, el problema de la posibilidad del conocimiento de la conducta se resuelve de manera racional, suponiendo que el comportamiento de los demás permanece fijo o inmóvil mientras se sigan reforzando las conductas esperadas. A este supuesto filosófico Hessen (2009) le llama dogmatismo. Se supone que, dado que los comportamientos se mantienen sin cambios, es posible conocerlos y transmitirlos de generación en generación.

Tal vez el sueño último del autor comentado sea controlar los comportamientos humanos de tal manera que toda la humanidad quede totalmente determinada: "Sirvámonos de nuestro poder para controlar, cada vez en aumento, a fin de crear unos individuos que no necesiten y que acaso no respondan ya al control” (Skinner, 1985, p. 239).

A manera de cierre - y como ya se comentó en el apartado anterior, con conocimiento no de hecho-, el conductista pretende conocer, predecir y controlar el comportamiento humano; intentando resolver los principales problemas filosóficos del conocimiento 


\section{Revista Iberoamericana \\ de las Ciencias Sociales y Humanísticas}

ISSN: $2395-7972$

(posibilidad, origen esencia-relación sujeto-objeto, existencia y composición de la realidad) de manera racional y la consecuencia necesaria de este hecho es que se ubica en supuestos filosóficos dogmáticos (posibilidad del conocimiento), racionales (origen del conocimiento), objetivistas (relación sujeto objeto de conocimiento), idealistas (existencia de la realidad) y dualistas (composición de la realidad), elaborando ideas, juicios y raciocinios de tipo racional o mental sobre la conducta humana con conceptos y criterios de verdad inmanentes. Es decir, por esa falta de orden en el comportamiento humano, como ya se comentó en el cierre del apartado anterior, el sujeto cognoscente de la teoría conductista tendrá que sustituir la certeza que tendría si realmente la conducta humana se pudiera conocer en esencia, por una suposición, consideración o estimación elaborada por su alma o razón para poner orden en el comportamiento de la humanidad.

\section{Discusión}

Los hallazgos revelan que las ideas, juicios y raciocinios que todo sujeto cognoscente elabora sobre cualquier fenómeno, dependen — de manera necesaria - de determinados supuestos filosóficos en los que se ubica, con conocimiento o no del fenómeno.

La relación de dependencia de la teoría conductista aplicada a la educación respecto de la filosofía — que se planteó como hipótesis general de este estudio- quedó demostrada en el sentido de que los supuestos filosóficos en los que se ubica la teoría dependen, de manera necesaria, de determinadas necesidades lógicas, ontológicas, epistemológicas y existenciales del sujeto cognoscente.

Este hecho ha sido ignorado a lo largo de la historia de la filosofía, incluso por los mismos epistemólogos. Hace más de un siglo Juan Hessen (1999) advirtió sobre el problema del conocimiento y de los supuestos con los que se pretende resolver, pero ignoró por completo que dependen de las necesidades lógicas, ontológicas, epistemológicas y existenciales del sujeto que pretende conocer, y ubicó su discurso - desconociendo este hecho - en una postura epistemológica dogmática (posibilidad de conocer), racional (origen del conocimiento), objetiva (relación sujeto objeto), idealista (existencia de la realidad) y dualista (composición de la realidad) construyendo una epistemología de tipo racionalista con conceptos y criterios inmanentes de verdad, y descuidando las otras posibilidades que se pueden obtener utilizando los sentidos o a partir de una mezcla entre estos y la razón. Supuso, 


\section{Revista Iberoamericana \\ de las Ciencias Sociales y Humanísticas}

ISSN: $2395-7972$

ingenuamente, que el conocimiento en esencia es posible, que nace en la razón, que es neutral, ideal y dualista, respectivamente.

Con John Locke (1994) sucedió todo lo contrario. Sostiene una epistemología escéptica, subjetivista, relativista y pragmática (posibilidad de conocer), empírica (origen del conocimiento), subjetiva (relación sujeto objeto), realista (existencia de la realidad) y monista (composición de la realidad) construyendo una epistemología de tipo sensual con conceptos y criterios trascendentes de verdad. Supuso, ingenuamente, que el conocimiento en esencia no es posible, que nace en los sentidos, que es interesado, real y monista, respectivamente.

Con Verneaux (2011) sucedió algo parecido. Siguiendo la epistemología de Aristóteles - que después fue recuperada por Santo Tomas de Aquino- construyó una epistemología de tipo crítico (posibilidad de conocer), intelectualista (origen del conocimiento), dialéctica (relación sujeto objeto), fenomenológica (existencia de la realidad) y pluralista (composición de la realidad) que permite la generación de discursos mixtos (sensual-racionales) con conceptos y criterios mixtos de verdad (intuitivo-racionales) y descuidando la riqueza de lo puramente racional, puramente empírico y lo racional-sensual.

Con Polo (2006) ocurrió todo lo contrario que con Verneaux. Siguiendo la tradición Kantiana, construyó una epistemología de tipo crítico (posibilidad de conocer), apriorista (origen del conocimiento), dialéctica (relación sujeto objeto), fenomenológica (existencia de la realidad) y pluralista (composición de la realidad) que permite la generación de discursos mixtos (racional-sensuales) con conceptos y criterios mixtos de verdad (racional-intuitivos) y descuidando la riqueza de lo puramente racional, puramente empírico y lo sensual-racional.

Los cuatro párrafos anteriores dan una idea sobre las cuatro posibilidades que el ser humano tiene para suponer que conoce en el arte, la ciencia, la religión y la misma filosofía: a partir de su razón, sus sentidos, su razón primero y después sus sentidos, y sus sentidos primero y después su razón. Es decir, conocimiento racional, sensual o mixto (sensitivoracional o racional sensitivo).

En este estudio se sostiene que ninguno de los cuatro es mejor que los otros y que todo depende del objeto de estudio y de los intereses lógicos, ontológicos, epistemológicos y existenciales del sujeto cognoscente. La ciencia no es neutral: obedece a intereses personales y/o de grupo. 


\section{Revista Iberoamericana de las Ciencias Sociales y Humanísticas}

ISSN: $2395-7972$

Los autores contemporáneos (Diez y Moulines, s. f.) no han aportado nada nuevo a la discusión epistemológica como se entiende en este estudio; lo que han hecho es adherirse a una de las cuatro posturas mencionadas confrontando sus ideas con los autores que defienden las otras posturas. Eso se puede observar en la infinidad de congresos que se llevan a cabo a nivel mundial. Se ha ignorado el hecho de que los problemas del conocimiento no tienen una única solución que permita la certeza en el conocimiento y que la misma es sustituida en los argumentos con determinados supuestos filosóficos que la mente del sujeto cognoscente elabora dependiendo de la facultad cognoscitiva con la que pretenda resolver los problemas del conocimiento que, a su vez, depende de sus intereses lógicos, epistemológicos, ontológicos y existenciales.

Sería bueno analizar, en los mismos términos de este estudio, las demás teorías que se aplican a la educación, como lo son el psicoanálisis, el humanismo, el cognoscitivismo, la psicogenética y la teoría sociocultural, etc., para tener una idea más clara de sus alcances y limitaciones epistemológicas, ontológicas, lógicas y existenciales, y de las relaciones entre estas y la filosofía, en el entendido de que difieren enormemente unas de otras.

Derivado de las conclusiones anteriores, se precisa de una formación sólida en epistemología durante los posgrados para que los futuros investigadores entiendan que la ciencia obedece a intereses que relacionan lo filosófico (epistemológico, ontológico, lógico y existencial) con lo teórico.

Algunas líneas de investigación que quedan pendientes para futuros estudios pueden ser las siguientes: la relación entre los supuestos filosóficos con los que se pretende resolver los problemas del conocimiento y los mismos problemas del conocimiento, entre los problemas del conocimiento y facultades cognoscitivas del sujeto cognoscente, entre las facultades cognoscitivas del sujeto cognoscente y los principios de la ciencia y entre los principios de la ciencia y las necesidades existenciales, lógicas, ontológicas y epistemológicas del sujeto cognoscente, etc. 


\section{Conclusiones}

Entre la filosofía y la teoría psicológica conductista aplicada a la educación es posible observar una relación de determinación de la primera sobre la segunda. Dicha dependencia del conductismo respecto de la filosofía se pude desglosar en una serie de relaciones menores que es posible establecer entre las entidades mencionadas: en primer lugar, las ideas, los juicios y los raciocinios que el sujeto cognoscente de la teoría conductista elabora sobre el comportamiento humano dependen, de manera necesaria, de los supuestos filosóficos con los que el sujeto cognoscente conductista pretenda resolver los principales problemas del conocimiento de la conducta.

El conductista pretende conocer, predecir y controlar el comportamiento humano intentando resolver los principales problemas filosóficos del conocimiento (posibilidad, origen, esencia-relación sujeto-objeto, existencia y composición de la realidad-, clasificación y concepto y criterio de verdad) con conocimiento o no del hecho- de manera racional, y la consecuencia necesaria de este suceso es que se ubica - también con conocimiento o no del hecho- en supuestos filosóficos dogmáticos (posibilidad del conocimiento), racionales (origen del conocimiento), objetivistas (relación sujeto objeto de conocimiento), idealistas (existencia de la realidad) y dualistas (composición de la realidad), elaborando ideas, juicios y raciocinios de tipo racional o mental sobre la conducta humana con conceptos y criterios de verdad inmanentes.

Debido esa falta de orden en el comportamiento humano, el sujeto cognoscente de la teoría conductista tendrá que sustituir la certeza que tendría si realmente la conducta humana se pudiera conocer en esencia, por una suposición, consideración o estimación elaborada por su alma o razón para poner orden en el comportamiento de la humanidad.

Es posible afirmar que el primer aspecto de la relación filosofía-teoría conductista se establece entre las ideas, juicios y raciocinios que el sujeto cognoscente de la teoría conductista elabora sobre el comportamiento humano y los supuestos filosóficos con los que pretende o intenta resolver los problemas del conocimiento de la conducta humana.

En segundo lugar, con conocimiento no del hecho, el conductista pretende conocer la conducta humana utilizando como facultad cognoscitiva su alma o razón pretendiendo resolver con ella los principales problemas filosóficos del conocimiento humano (posibilidad, origen, esencia — relación sujeto-objeto, existencia y composición de la 


\section{Revista Iberoamericana \\ de las Ciencias Sociales y Humanísticas}

ISSN: $2395-7972$

realidad- clasificación y concepto y criterio de verdad) debido a la necesidad existencial de predecirla y controlarla; es decir, de poner orden en el comportamiento humano.

Se presume que el segundo aspecto de la relación filosofía-teoría conductista queda señalado entre los supuestos filosóficos con los que el sujeto cognoscente de la teoría conductista pretende resolver los problemas del conocimiento del comportamiento humano y la facultad cognoscitiva que utiliza para ello: en este caso, su razón, alma, mente, espíritu, inteligencia o como se le quiera llamar a ese ente con el que pensamos.

En tercer lugar, ante la imposibilidad del sujeto cognoscente de conocer - en esencia- el comportamiento humano debido quizá a que carece de las facultades cognoscitivas para ello; este se debe conformar con suponerlo, conjeturarlo, presumirlo, creerlo, sospecharlo o admitirlo a partir de determinados supuestos filosóficos con los que pretende resolver los problemas filosóficos del conocimiento del comportamiento humano.

Se estima que el tercer aspecto de la relación filosofía-teoría conductista se puede fijar entre los problemas filosóficos del conocimiento de la conducta humana (posibilidad, origen, esencia -relación sujeto-objeto de conocimiento, existencia y composición de la realidad conductual-, clasificación y concepto y criterio de verdad), y los respectivos supuestos filosóficos (dogmatismo, racionalismo, objetivismo, idealismo, dualismo, etc.) ya mencionados, con los que el sujeto cognoscente de la teoría conductista pretende resolverlos.

En cuarto lugar, para elaborar ideas, juicios y raciocinios sobre el comportamiento humano, nuevamente - con conocimiento o no del hecho- el sujeto cognoscente de la teoría conductista parte de determinados principios de la ciencia que se relacionan con los supuestos filosóficos ya mencionados, con los que pretende resolver los problemas del conocimiento de la conducta humana, utilizando para ello como facultad cognoscitiva su razón o alma.

Los principios lógicos (no contradicción, identidad, tercero excluido, razón suficiente y causalidad) a partir de los cuales el sujeto cognoscente de la teoría conductista pretende expresar las razones en que se apoyan los raciocinios sobre el ser, es decir, sobre el comportamiento humano, se relacionan con los supuestos con los que intenta resolver los problemas filosóficos del conocimiento de la conducta, utilizando como facultades cognoscitivas su alma o razón. 


\section{Revista Iberoamericana de las Ciencias Sociales y Humanísticas}

ISSN: $2395-7972$

Los principios ontológicos (reposo o quietud, determinismo, exclusión, seguridad y determinismo, respectivamente) a partir de los cuales el sujeto cognoscente de la teoría conductista pretende transformar el ente (el comportamiento) en ser (conocer, predecir y controlar la conducta), se relacionan con los supuestos filosóficos con los que intenta resolver los problemas filosóficos del conocimiento de la conducta, utilizando como facultades cognoscitivas su alma o razón.

Los principios epistemológicos o existenciales (predicción, manipulación, control, etc.) a partir de los cuales el sujeto cognoscente de la teoría conductista pretende conocer, predecir y controlar la conducta humana, se relacionan con los supuestos filosóficos con los que intenta resolver los problemas filosóficos del conocimiento de la conducta, utilizando como facultad cognoscitiva su alma o razón.

Es de conjeturar que el cuarto aspecto de la relación filosofía-teoría conductista se puede fundar entre los supuestos filosóficos con los que el sujeto cognoscente pretende resolver los problemas del conocimiento de la conducta humana, utilizando como facultad cognoscitiva su razón o alma, y los principios de la ciencia con los que pretende elaborar ideas, juicios y raciocinios sobre el comportamiento humano.

Finalmente, en quinto último lugar, es de esperarse que los principios de la ciencia (lógicos, ontológicos y epistemológicos) que el sujeto cognoscente utilice para elaborar ideas, juicios y raciocinios sobre el comportamiento humano, se relacionan con sus necesidades existenciales (alimentación, casa, vestido, educación, recreación, etc.). Esto lo obliga, en cierto sentido, a resolver los problemas filosóficos del conocimiento utilizando para ello como facultad cognoscitiva su razón o espíritu y ubicándose, con conocimiento o no del hecho, en determinados supuestos filosóficos con los que pretende solucionar los problemas del conocimiento.

Es posible considerar que el sexto aspecto de la relación filosofía-teoría conductista se puede establecer ente los principios de la ciencia (lógicos, ontológicos, epistemológicos) y las necesidades existenciales (alimentos, ropa, habitación, educación, recreación, etc.) del sujeto cognoscente de la teoría conductista.

En última instancia, el motor que promueve los principios de la ciencia o del conocimiento que se utilizan para elaborar los discursos de la teoría conductista son las necesidades existenciales, epistemológicas, ontológicas y lógicas del sujeto cognoscente dependiendo de circunstancias de modo, tiempo y lugar. 
Revista Iberoamericana

de las Ciencias Sociales y

Humanísticas

ISSN: $2395-7972$

En ese entendido, se puede sugerir una relación o correspondencia entre la filosofía y la teoría conductista en términos de necesidades existenciales, epistemológicas, ontológicas y lógicas del sujeto cognoscente en determinado tiempo, lugar y circunstancias.

Lo anterior permite concluir, entre otras cosas, que el mapeo del concepto mencionado con la voz conductismo se establece en términos de alguna definición de la noción mentada con la voz filosofía que determina la utilización de ciertos principios del conocimiento o de la ciencia dependiendo de las necesidades lógicas, ontológicas, epistemológicas y existenciales del sujeto cognoscente de la teoría conductista en determinadas circunstancias de modo, tiempo y lugar.

\section{Futuras líneas de investigación}

Los aspectos más importantes de la relación filosofía-teoría que se destacaron en este trabajo, y que son potencialmente muy interesantes para desarrollos posteriores, se refieren a las relaciones entre necesidades existenciales y necesidades ontológicas, entre necesidades ontológicas y problemas del conocimiento, entre problemas del conocimiento y supuestos filosóficos, entre supuestos filosóficos y facultades cognoscitivas, entre facultades cognoscitivas y principios de la ciencia, entre otras relaciones no menos importantes que serán motivo de nuevas investigaciones con motivo de relacionarlos con las demás teorías que se aplican a la educación, como lo son el psicoanálisis, el humanismo, el cognoscitivismo, la psicogenética y la teoría sociocultural, etc., para tener una idea más clara de sus alcances y limitaciones epistemológicas, ontológicas, lógicas y existenciales, y de las relaciones entre estas y la filosofía, en el entendido de que difieren enormemente unas de otras; investigaciones, todas ellas, que permitirían la conformación de un libro que podría nombrarse: "Filosofía y teoría educativa. Una relación onto-epistemológica". 
Revista Iberoamericana

de las Ciencias Sociales y

Humanísticas

ISSN: 2395 - 7972

\section{Referencias}

Aristóteles (1992). Metafísica. México: Porrúa.

Bergson, H. (1986). Introducción a la metafísica. México: Porrúa.

Diez, J. A. y Moulines, U. (s. f.). Fundamentos de filosofía de la ciencia. Barcelona: Ariel.

Garrigou-Lagrange, R. (1980). El sentido común. La filosofía del ser y las formas dogmáticas. Madrid: Ediciones Palabra.

Gutiérrez, R. (2005). Introducción a la lógica. México: ESFINGE.

Guzmán, J. C. y Hernández Rojas, G. (1993). Implicaciones educativas de seis teorías psicológicas. México: CONALTE.

Heidegger, M. (2006) ¿Qué es la filosofía? España: Herder.

Hessen, J. (2009). Teoría del conocimiento. México: Porrúa.

Locke, J. (1994). Ensayo sobre el entendimiento humano. México: GERNIKA.

Natorp, P. (1987). Propedéutica filosófica. México: Porrúa.

Ortega y Gasset, J. (1998). ¿Qué es filosofía? México: Porrúa.

Polo, L. (2006). Curso de teoría del conocimiento (tomo I). Pamplona, España: Ediciones Universidad de Navarra, S. A.

Schopenhauer, A. (1997). El mundo como voluntad y representación. México: Porrúa.

Skinner, B. F. (1985). Aprendizaje y comportamiento. España: Ediciones Martínez Roca, S.

A.

Skinner, B. F. (1986). Más allá de la libertad y la dignidad. España: Ediciones Martínez Roca, S. A.

Skinner, B. F. (1994). Sobre el conductismo. España: Psico-libro.

Simmel, G. (1946). Problemas fundamentales de la filosofía. Madrid, España: Revista de Occidente.

Verneaux, R. (2011). Curso de filosofía tomista. Epistemología general o crítica del conocimiento. España: Herder. 